\title{
Nouveaux défis, nouvelles démarches comparatives
}

Christoph Kodron

\section{(2) OpenEdition}

1 Journals

Édition électronique

URL : http://journals.openedition.org/ries/4308

DOI : 10.4000/ries.4308

ISSN : 2261-4265

Éditeur

Centre international d'études pédagogiques

Édition imprimée

Date de publication : 1 mars 1994

Pagination : 87-95

ISSN : 1254-4590

\section{Référence électronique}

Christoph Kodron, « Nouveaux défis, nouvelles démarches comparatives », Revue internationale

d'éducation de Sèvres [En ligne], 01 | 1994, mis en ligne le 20 avril 2015, consulté le 22 avril 2019. URL http://journals.openedition.org/ries/4308; DOI : 10.4000/ries.4308

Ce document a été généré automatiquement le 22 avril 2019.

(c) Tous droits réservés 


\title{
Nouveaux défis, nouvelles démarches comparatives
}

\author{
Christoph Kodron
}

1 Je me bornerai à évoquer le secteur de l'éducation, et plus précisément encore celui de l'éducation scolaire. Je ne traiterai ni de comparaison internationale ni d'éducation comparée, en tant que discipline, car mon objectif est de montrer que les nouveaux défis du monde moderne nous imposent de développer systématiquement dans tous les secteurs de l'éducation une culture quotidienne de la comparaison.

\section{Le monde entier rapetisse, et l'Europe plus encore}

2 Depuis des années, on parle de global village pour résumer les effets d'interdépendance induits par les conséquences de la technologie atomique, de la pollution, par la menace de changements climatiques, le souci de préserver les ressources naturelles de l'homme. Ces interdépendances ont révélé l'impossibilité, pour une région ou un pays, d'une survie en solitaire. De plus en plus d'activités humaines tendent à avoir des implications pour le monde entier. Le secteur de l'éducation scolaire ne saurait échapper à des changements d'une aussi grande ampleur.

3 Les nouvelles technologies des médias et de la communication, les moyens de transport, ainsi que la mobilité des populations sont en train de définir et de construire des espaces infiniment plus vastes que ceux auxquels nos États nationaux européens restent confinés. Les progrès vers la construction d'un espace européen, du fait, surtout, de l'Union européenne, progrès encore accélérés par la chute du système socialiste et l'ouverture de ces pays vers l'Ouest, ont déjà suscité des échanges, des coopérations et aussi des coproductions dont nos parents n'auraient pas même osé rêver, lorsqu'ils parlaient, juste après la guerre, de réconciliation en Europe. Je pense à l'accroissement énorme des échanges commerciaux, des biens et des services, du tourisme et des migrations, mais aussi à l'échange culturel induit par les médias, dont on perçoit déjà l'influence notamment sur les coutumes, la consommation, les habitudes alimentaires. 
Des coopérations existent déjà depuis le niveau des entreprises multinationales jusqu'à celui de l'artisanat, avec l'échange de stagiaires, en formation initiale, comme en formation continue. Dans l'Union européenne, et en partie aussi au niveau du Conseil de l'Europe, une reconnaissance formelle des formations, des certificats et des diplômes est déjà acquise, même si la pratique est hérissée de difficultés et d'obstacles, quand elle ne se heurte pas au refus ou à la condescendance.

On n'aura garde d'oublier la nécessité politique de rapprocher les gens de cultures et de langues différentes. C'est une évidence en Europe, parce que la politique au niveau des gouvernants et l'économie à elles seules ne feront pas naître, à moyen terme, un espace européen sans qu'éclatent des conflits sanglants. Les systèmes éducatifs, avec leurs 50 millions d'élèves devront y contribuer par leur ouverture et leur impact.

\section{Nos écoles empruntent-elles cette direction nouvelle?}

6 Si l'on pose en principe que l'Europe doit être une référence importante, où en est-on en matière d'éducation? Nos systèmes d'éducation sont-ils prêts à coopérer, en tant que systèmes, ou bien continuent-ils dans la direction prise au dix-neuvième siècle, lorsqu'ils étaient pour l'État l'outil privilégié de constitution d'un système homogène, national ? Le système éducatif n'a-t-il pas contribué à construire dans les têtes les frontières nationales et culturelles, par le biais d'une orientation nationaliste, par la glorification des histoires nationales, par la transmission d'une culture nationale unifiée, définie nationalement, par des diplômes nationaux? Nos systèmes éducatifs se sont-ils ouverts au voisin, aux petits peuples, aux autres Européens et, finalement, à l'espace mondial ? Les connaissances que l'école cherche à transmettre, les savoir-faire, les attitudes et les valeurs sont-ils adaptés à ces nouvelles demandes, aux nouvelles réalités que nous vivons déjà et que nos enfants vivront avec plus d'intensité encore ? Peut-on dire que la comparaison, comme démarche fondamentale pour promouvoir cette réorientation, est déjà entrée dans les pratiques ? Je suis persuadé que non!

\section{La comparaison comme un outil de réorientation}

7 Il convient de se demander, au préalable, quel peut être en ce domaine l'apport de la recherche comparative des sciences de l'éducation et, plus spécialement, de l'éducation comparée. A-t-elle fourni les méthodes et les informations nécessaires pour que s'opère semblable réorientation en direction de l'ouverture et de la collaboration? Il ne s'agit pas là de problèmes qui relèvent du niveau de la science ${ }^{1}$ mais plutôt des effets constatés à un autre échelon, celui des comparaisons à exercer au quotidien. Porter son regard par-delà les frontières est du ressort traditionnel de l'éducation comparée. Les comparaisons traitent deux ou plusieurs systèmes sous l'angle de questions systématiquement posées. On rencontre aussi des travaux où la comparaison reste implicite : un autre système est décrit à partir d'un système de référence, sans que celle-ci soit explicite. À mon sens, on ne reconnaît pas encore suffisamment la validité de bonnes descriptions fondées sur la visite d'un pays étranger. Même si la comparaison reste implicite, la lisibilité d'une bonne description, ainsi que la richesse des aspects traités, continuent à justifier largement de telles enquêtes. 
8 La curiosité du regard est au principe de toute visée passant par-dessus les limites traditionnelles; elle est indispensable pour toute ouverture. L'ouverture elle-même, et la collaboration plus encore, exigent que l'on se situe soi-même dans son rapport à l'autre, à l'étranger. Peu importe le but que l'on assigne à sa comparaison, la question posée ou le thème à traiter : c'est son propre système qui restera toujours la référence majeure, et c'est sur cette base qu'on effectuera la comparaison avec l'étranger. Mais chaque comparaison a comme effet que ce qui était étranger devient plus familier et plus compréhensible dans son étrangeté même.

9 Les études comparatives destinées au public spécialisé qu'intéresse l'éducation ont connu une augmentation considérable depuis que le grand public s'est mis à se préoccuper de l'Europe et que l'éducation est apparue comme un facteur décisif dans la concurrence économique internationale. Mais la plupart de ces travaux adoptent un point de vue trop élevé, celui, par exemple, du planificateur ministériel traitant d'un ou de plusieurs systèmes éducatifs, tandis que d'autres en restent au niveau des systèmes. Ces travaux sont assurément nécessaires, mais ils n'aident pas vraiment à comprendre un autre système dans son fonctionnement interne, ne permettent pas d'approcher les domaines qui sont précisément imprégnés par une culture spécifique. Quand on demande aux administrateurs, aux constructeurs de programmes scolaires, aux enseignants, aux parents, ce qu'ils ont pu tirer des perspectives, ainsi dessinées d'en haut, pour répondre, dans leurs tâches quotidiennes, aux nouveaux défis, les réponses témoignent de leur insatisfaction!

\section{Notre réalité européenne est difficile à comparer}

Les bibliographies font apparaître une grande quantité de publications constituées par des travaux comparatifs sur certains aspects des systèmes de l'éducation (ou plutôt de scolarisation) et de formation en Europe. Mais comme beaucoup de ces travaux dépendent d'institutions supranationales - comme l'OCDE, l'Union européenne, le Conseil de l'Europe - ou des ministères nationaux, ils fondent leurs analyses sur les systèmes tels qu'ils sont définis par les États membres. Pour mon cas personnel, la République fédérale d'Allemagne, cela pose déjà un problème. Souvent, dans les analyses sur le système d'éducation faites à ce niveau supranational, figure The case of Germany, alors que, dans notre système fédéral, les seize Länder sont souverains pour la politique culturelle qui inclut le système scolaire et la formation des enseignants. On trouve souvent, à titre d'exemple, l'un des seize Länder, ou encore un texte qui compile déjà une comparaison des ressemblances au niveau « national ».

11 Au niveau européen, il est difficile de dire combien de systèmes scolaires coexistent. Tout dépend de la perspective adoptée. Soit on respecte le cadre de l'État (Espagne, France, Italie etc.), soit on privilégie la responsabilité ministérielle (par exemple, le ministère de la culture de Hesse), soit encore on part des droits spécifiques (l'Alsace en France, la Galice en Espagne) ou de celui des minorités qui ont des spécificités quant à leur système scolaire ou leurs écoles (ainsi les Slovènes en Italie, les Souabes en Saxe).

Une autre constatation s'impose : on ne dispose toujours pas d'une terminologie adéquate pour comparer ces réalités diverses de l'éducation dans une perspective européenne. Celle-ci doit impliquer en même temps trois niveaux :

- la comparaison entre les systèmes d'éducation nationaux ; 
- la comparaison de ce qui est établi pour les minorités territoriales nationales ${ }^{2}$ et les minorités de migrants dans les pays ;

- la comparaison de la scolarisation des immigrants ${ }^{3}$, les nouvelles minorités récemment installées. plus aux seuls politiciens et administrateurs à pratiquer certaines formes de coopération et de comparaison pour leur planification. Tous les niveaux d'un système d'éducation s'ouvrent, commencent à coopérer et, ce faisant, pratiquent aussi des comparaisons. Mais nous sommes tous «naturellement » pris dans notre système d'éducation, établi depuis longtemps, avec ses coutumes et ses traditions qui en ont façonné les structures, qui ont abouti à des définitions et surtout des connotations qui nous semblent «naturelles». Au niveau du programme, nous parlons depuis longtemps du hidden curriculum, de ce qui se transmet d'une manière "naturelle», sans que cela soit prescrit par des textes (programmes) ni même formulé explicitement. Le même phénomène se retrouve dans tous les secteurs de n'importe quel système éducatif ou scolaire. Dans notre propre société, dans notre culture, nous éprouvons déjà des difficultés à expliquer le système scolaire dans ses structures et son fonctionnement officiel. Il est encore plus difficile de démontrer explicitement ce qui se fait et qui est caché par la tradition, dissimulé dans les structures et les coutumes. Il est encore plus difficile d'expliquer le système d'éducation avec tous ses facteurs sociaux et tous les acteurs impliqués. Très souvent nous expliquons la réalité actuelle à l'aide de notre expérience personnelle, qui est liée au niveau social de nos parents, à notre propre - et lointaine - expérience scolaire, à notre formation professionnelle, à nos orientations politiques, nos fonctions présentes, etc.

Quand un non-spécialiste - même un enseignant - essaie d'expliquer son propre système à un étranger, il constate généralement qu'on le connaît fort peu, que ce soit dans ses grandes lignes ou dans ses détails. Il prend conscience de ce que les explications qu'il fournit ne sont pas fondées sur une information exacte et actualisée, mais sur une masse de données rassemblées un peu au hasard, sans connaissance véritable. Bien des réponses s'appuient sur le sentiment vague que les choses sont ou doivent être ainsi ${ }^{4}$. Il n'a ainsi du système scolaire qu'une vision générale, quasi mythique, dans sa globalité et seulement 
un peu détaillée dans le secteur spécifique où il travaille. À certaines questions posées par un étranger, il ne sait quoi répondre, soit parce qu'elles touchent à un champ mal connu, soit parce qu'il peut seulement expliquer sa pratique, tout en en ignorant les causes ou l'histoire. Il est possible aussi qu'il n'ait pas vraiment compris la question. Par exemple, présent, comme personne-ressource, à un congrès français sur «la relation écoleentreprise", je n'ai compris des questions telles que "Qui paie les coûts des stages d'entreprise? » qu'après deux essais infructueux de réponse, parce que la question visait les coûts pour le personnel de l'entreprise s'occupant d'un stagiaire. Une telle question ne se poserait jamais en RFA, où c'est toujours le patronat qui a voulu les stages et s'en sent responsable.

17 Quand et où avons-nous appris à expliquer notre système, ses fonctions, ses formes ? Avons-nous, dans notre éducation, systématiquement appris à présenter notre système d'éducation à un étranger qui n'a pas la même culture et qui ne parle pas la même langue ${ }^{5}$ ? On ne trouve rien de tel dans les programmes scolaires, malgré la demande d'intégrer la « dimension européenne » formulée par les ministres de l'éducation en $1988^{6}$. Aussi, au niveau universitaire, l'exercice de la comparaison est-il plutôt rare. Pour bien comparer, il faudrait pratiquer un double changement de perspective : observer son propre système et se mettre à la place d'un étranger ${ }^{7}$.

ll nous manque un guide, une méthode, pour nous approprier la réalité d'un système étranger, et aussi une formation à la comparaison pour ceux qui travailleront en éducation.

\section{De la difficulté de s'approprier la réalité d'un système scolaire}

Un étranger qui s'efforce de s'approprier un système étranger se trouve fondamentalement dans la même situation, mais se heurte à plus de problèmes encore. Lui aussi se base, de la même façon, sur ses connaissances et la vision qu'il a de son propre système quand il s'approche d'un système étranger. Souvent, il s'intéresse surtout au secteur de l'éducation qu'il connaît du fait de son propre travail. Il ne pose que des questions induites par le fonctionnement du système qu'il connaît. Il serait souhaitable et fructueux d'essayer de comprendre en premier lieu le système étranger dans ses grandes lignes et selon son propre fonctionnement, avant de poser les questions que l'on apporte et par lesquelles on est au premier chef intéressé.

20 Mais, même avec la meilleure volonté, des difficultés subsisteront. Elles tiennent à l'habitude de présenter son système, à la manière d'en tracer les grandes lignes, à ce qu'on explique et à ce qu'on ne dit pas. Cette présentation inclut toujours une partie culturellement marquée, liée à la culture sociale et au système politique et une autre partie, individuelle, dépendante cette fois de la formation.

21 J'illustrerai ce point par un exemple. Au début de mon travail comparatif, j'ai eu la chance d'effectuer une visite approfondie dans une école normale de l'Est de la France avec un petit groupe d'Allemands venus de différents Länder. D'abord, la directrice nous a reçus pour nous expliquer le système de formation des maîtres, le statut et le fonctionnement des Écoles normales, le cursus et le contenu de la formation des élèves-maîtres, avant de nous montrer les locaux et les salles. Elle nous a brossé un tableau bien structuré, en mettant l'accent sur le fait que toutes les écoles normales fonctionnent de la même façon 
et sur la base des textes communs. En visitant les salles, j'ai vu, par hasard, qu'un enseignement de la religion était indiqué pour une salle. Ma demande d'explication reçut comme réponse que c'était là "une exception", mais que les écoles normales fonctionnaient partout en France à l'identique. C'est seulement de retour en Allemagne que je me suis rendu compte qu'il y avait au moins une autre différence : l'enseignement de l'allemand dans les zones «dialectophones». Peu après, je me suis trouvé, avec un groupe de participants à une conférence internationale, à étudier la formation des enseignants dans deux Länder de la RFA. C'est l'inverse qui s'est produit. Les responsables du système universitaire de la première phase de formation des maîtres nous ont expliqué que, dans leur institution, la formation se distinguait nettement de celle de l'autre Land, bien que les structures globales de formation fussent les mêmes. Les Français ont ainsi tendance à souligner l'unité du système national, même si un étranger s'intéresse surtout à ce qui est spécifique, tandis qu'en Allemagne, compte tenu de notre système fédéral ancien, nous sommes portés à privilégier les particularités, avant d'avouer qu'au fond, il y a, peut-être, un système fortement homogène, du fait des traditions communes, des concertations au niveau de la Conférence permanente des ministres de l'éducation des Lander et certaines lois-cadres de la Fédération. Des habitudes analogues se retrouveraient dans toutes les cultures et ne se révèlent normalement au comparatiste qu'après une enquête approfondie.

\section{Tirons davantage profit de ceux qui ont vécu un autre système}

22 Je suis persuadé que ni les professionnels de la comparaison, ni les enseignants ${ }^{8}$ ne pourront répondre seuls aux exigences d'une pratique éducative qui chercherait l'ouverture et la coopération. On ne pourra pas toujours accéder à la dimension comparative dans des écrits, ni l'explorer par des études individuelles incluant voyages et recherches, même si cela reste souhaitable. Une solution pratique et économique consisterait à profiter de ceux qui ont une expérience vécue d'un autre système, qui ont pratiqué une comparaison! Partout en Europe se rencontrent des gens qui viennent d'un autre pays, qui ont temporairement vécu dans un autre système scolaire, qui ont des expériences d'échanges. On pourrait leur demander des descriptions écrites ou orales sur un thème spécifique, si leurs connaissances sont approfondies, et les utiliser systématiquement comme personnes-ressources.

Je vois là une tâche commune que pourraient exécuter en collaboration professionnels de la comparaison ${ }^{9}$ et enseignants, afin de développer des méthodes pratiques et de les introduire dans l'enseignement, par exemple en formation continue. Il s'agirait d'explorer toutes les possibilités et de les transformer en une méthodologie d'enseignement de la comparaison, de comparer en enseignant, en tirant profit des personnes disponibles. Il faut développer leur expérience, leur poser les questions auxquelles elles pourront répondre et qui cerneront les spécificités du système étranger. 


\section{La coopération comme occasion d'une comparaison active} coopérer directement avec une institution d'un autre pays ${ }^{10}$. Cela est possible dans une coopération pédagogique qui échange informations, points de vue et matériel produit par les élèves. Il s'agit, là aussi, de développer des méthodes d'enseignement utilisant systématiquement le dispositif pédagogique que chaque groupe doit élaborer pour l'autre (la perspective sur soi-même, sur ce qui vous est propre) et de traiter les informations, les productions reçues de l'autre (la perspective de l'étranger). Des coopérations multilatérales sont également déjà pratiquées ${ }^{11}$. Du point de vue de la comparaison, chacune des deux démarches a des avantages et des inconvénients. Dans le cadre d'une coopération bilatérale il est plus facile de pousser certaines questions de comparaison plus loin que dans les coopérations multilatérales. Mais on risque de verser dans des préjugés sur l'autre culture, lorsqu'on constate une différence culturelle. Dans des coopérations multilatérales, on court moins le risque de tirer des conclusions erronées sur l'autre, sa culture et ses pratiques. Le fait qu'il y ait plusieurs partenaires aboutit à ce qu'il y ait le plus souvent quelqu'un qui agit de même. Mais l'intégration de plusieurs partenaires dans la démarche amène aussi à rester davantage à la surface des phénomènes et des faits.

25

Ceux qui ont pratiqué la recherche comparative internationale en éducation ont produit des comparaisons nécessaires et utiles, ils ont aussi développé un vaste éventail de méthodes scientifiques. Mais, dans la perspective des nouveaux défis pour tous les secteurs de l'éducation, surtout en Europe, il faut sortir de la tour d'ivoire académique et populariser les connaissances, les rendre plus accessibles, et élargir aussi les méthodes de comparaison. De plus, une coopération intensive entre les praticiens de l'éducation et les comparatistes est indispensable pour développer des méthodes de comparaisons adaptées aux différents niveaux de l'enseignement et aux différents partenaires. Essayons de développer un enseignement à la comparaison et des comparaisons à enseigner pour mieux nous comprendre en Europe et au-delà.

\section{NOTES}

1. La recherche scientifique comparative en éducation comporte deux axes. Le premier est celui des chercheurs d'universités et des centres de recherche scientifique. Le second tourne autour des institutions politiques internationales, comme le BIE, la Banque mondiale, l'OCDE, l'Union européenne. Mais les recherches effectuées tendent toutes à s'adresser prioritairement aux spécialistes. De plus, il n'est pas toujours facile de se procurer des textes qu'on trouve rarement dans les librairies générales et qui sont difficiles à acheter.

2. Une comparaison de la différence de traitement est instructive : les Frisons vivant dans la province de Frise aux Pays-Bas ont le droit de pratiquer leur langue à l'école, dans

Revue internationale d'éducation de Sèvres, 01 | 2015 
l'administration et en public ; ceux qui vivent de l'autre côté de la frontière en RFA n'ont qu'un dialecte qui n'a pas de statut officiel. Les Basques vivant au Pays basque espagnol ont le droit de mettre leurs enfants dans des écoles où le basque est langue d'enseignement ; ceux qui vivent de l'autre côté de la frontière en France ont peut-être la chance que leur langue soit enseignée comme « langue régionale».

3. II faut se souvenir qu'il y a 5 millions de citoyens européens qui vivent dans un pays de l'UE dont ils n'ont pas la nationalité. On bute aussi sur une difficulté de langue : d'un point de vue, ce sont des citoyens européens depuis l'Acte unique et encore plus après Maastricht ; mais pour les pays membres ce sont des «étrangers » ou des «immigrés»; dans les textes de l'UE ce sont des « migrants».

4. II faudrait par exemple expliquer le fait qu'en France les parents s'efforcent de mettre leur enfant le plus tôt possible à l'école primaire, alors qu'en RFA l'âge moyen d'entrée à l'école primaire est à 6 ans et 8 mois, mais que, dans les deux pays, l'âge de la scolarité obligatoire est le même.

5. Voir, pour une démarche qui met «l'étranger" au centre: C. Alix, Pakt mit der Fremdheit? Interkulturelles Lernen als dialogisches Lernen im Kontext internationaler Schulkooperation, Frankfurt am Main, IKO-Verlag, 1990.

6. Beaucoup plus positivement: R. Ryba, «Towards an European Dimension in Education: Intention and Reality in European Community Policy and Practice ", Comparative Education Review, vol. 36 (1992), n 1, p. 10-24.

7. Cela fait aussi défaut dans la formation des enseignants! Dans Le français dans le monde, (série Recherche et application) va paraître au printemps 1994 un recueil intitulé « Vers une pédagogie des échanges » où beaucoup de ces questions seront traitées. Il me semble fort utile d'introduire systématiquement un changement de perspective dans la formation d'enseignants. En RFA, cela n'existe en principe que dans la formation des juristes.

8. Je suis convaincu qu'il faudrait introduire une formation à la comparaison, une part d'éducation comparée, dans la formation de tous les personnels d'enseignement en Europe. Mais, même si cela se réalisait, des "intervenants extérieurs", des personnes-ressources seront toujours nécessaires.

9. Je ne pense pas seulement aux chercheurs en éducation comparée ou aux universitaires travaillant à des comparaisons, mais aussi, dans le monde du travail, à ceux qui ont fait des comparaisons ou ont eu la pratique de différents systèmes, qui connaissent, par exemple, les différences de structure hiérarchique des entreprises, les différences dans les techniques de vente de certains produits, etc.

10. Voir, sur une telle démarche tendant à développer un enseignement comparatif: C. Alix, C. Kodron, «Coopérer et se comprendre, propositions pour une conception dialogique et de la rencontre, pour une pédagogie de la communication interculturelle centrée sur un thème, pour une coopération entre établissements scolaires de différents pays » (document de travail), Paris, Office franco-allemand de la jeunesse, 1988.

11. «Bilan et perspectives des échanges scolaires", Barcelone, 13-17 mars 1991, Actes du colloque CECA-CEE-CEEA, Task Force Ressources Humaines Education, Formation, Jeunesse : Bruxelles 1991 (TFRH / 312-91). Seuls, actuellement, les échanges multilatéraux peuvent être subventionnés par l'UE. Un moyen économique consiste à utiliser l'e-mail: R. Keep, On-Line, Electronic, Mail in the Curriculum. NCET, University of Warwick Science Park, Coventry 1991. 


\section{RÉSUMÉS}

L'auteur propose de sortir de la tour d'ivoire académique et de populariser les connaissances, les rendre plus accessibles. De ce point de vue, il suggère d'intensifier la coopération entre les praticiens de l'éducation et les comparatistes.

INDEX

Index géographique : Europe

Mots-clés : coopération internationale, éducation comparée, enseignant, politique éducative, recherche comparative, système scolaire

\section{AUTEUR}

\section{CHRISTOPH KODRON}

Institut allemand de recherche pédagogique internationale (DIPFF), Francfort. 